\title{
High performance photolithographically-patterned polymer thin-film transistors gated with an ionic liquid/poly(ionic liquid) blend ion gel
}

\author{
Q. Thiburce, ${ }^{1}$ L. Porcarelli, ${ }^{2}$ D. Mecerreyes, ${ }^{2,3}$ and A. J. Campbell ${ }^{1,4, a)}$ \\ 1) Experimental Solid State Physics group, Department of Physics, Imperial College London, Blackett Laboratory, \\ South Kensington Campus, London SW7 2AZ, United Kingdom \\ ${ }^{2)}$ Institute for Polymer Materials, POLYMAT, University of the Basque Country (UPV-EHU), \\ Joxe Mari Korta Center, Avda. Tolosa 72, 20018 Donostia-San Sebastian, Spain \\ 3) IKERBASQUE, Basque Foundation for Science, E-48011 Bilbao, Spain \\ 4) Centre for Plastic Electronics, Imperial College London, South Kensington Campus, London SW7 2AZ, \\ United Kingdom
}

(Dated: 26 May 2017)

We demonstrate the fabrication of polymer thin-film transistors gated with an ion gel electrolyte made of the blend of an ionic liquid and a polymerised ionic liquid. The ion gel exhibits a high stability and ionic conductivity, combined with facile processing by simple drop-casting from solution. In order to avoid parasitic effects such as high hysteresis, high off-currents and slow switching, a fluorinated photoresist is employed in order to enable high-resolution orthogonal patterning of the polymer semiconductor over an area that precisely defines the transistor channel. The resulting devices exhibit excellent characteristics, with an on/off ratio of $10^{6}$, low hysteresis and a very large transconductance of $3 \mathrm{mS}$. We show that this high transconductance value is mostly the result of ions penetrating the polymer film and doping the entire volume of the semiconductor, yielding an effective capacitance per unit area of about $200 \mu \mathrm{F} \mathrm{cm}^{-2}$, one order of magnitude higher than the double layer capacitance of the ion gel. This results in channel currents larger than $1 \mathrm{~mA}$ at an applied gate bias of only - $1 \mathrm{~V}$. We also investigate the dynamic performance of the devices and obtain a switching time of $20 \mathrm{~ms}$, which is mostly limited by the overlap capacitance between the ion gel and the source and drain contacts.

Electrolyte-gated organic thin-film transistors (OTFTs) have received a lot of attention over the past years, thanks to their ability to operate at very low voltages $(<|1| \mathrm{V}),{ }^{1-3}$ which makes them particularly attractive for use in portable devices powered by low supply voltage sources such as thin-film batteries, and interfacing with conventional Si CMOS electronics. If an electrolyte is placed in contact with two electrodes, to which a small voltage is applied, ionic species within the electrolyte migrate towards the electrode of opposite polarity, forming ultra-thin electrical double layers at both electrolyte/electrode interfaces. When one of these electrodes is the channel of a transistor, large charge densities arise within the semiconductor, as a result of the ionic accumulation within a small volume close to the interface or within the bulk of the semiconductor, depending on the mode of operation of the device. In the case where ionic charges are contained at the interface with the semiconductor, for example by using a polyelectrolyte in which the ionic charges are covalently linked to the polymer chains ${ }^{4}$ or a crystalline semiconductor impermeable to ion penetration, ${ }^{5}$ the device operates much like a field-effect transistor. On the other hand, when ions penetrate inside the bulk of a polymer semiconductor, the capacitance must be considered as a volumetric parameter and the doping process is electrochemical in nature, ${ }^{6-8}$ resulting in very large currents flowing through the whole channel volume. As

a)Electronic mail: alasdair.campbell@imperial.ac.uk a consequence, large transconductances surpassing that of silicon-based TFTs can be obtained, ${ }^{9}$ even in OTFTs employing polymer semiconductors with modest charge carrier mobilities. This makes electrolyte-gated OTFTs suitable candidates for chemical and biomedical sensing applications, ${ }^{10,11}$ or to drive organic light-emitting diodes. $^{12}$

Amongst the variety of electrolyte materials, ion gels are an attractive class of solid electrolytes. ${ }^{13}$ They consist in the mixture of an ionic liquid (IL) - a salt that is in the liquid state at room temperature - contained within a polymer matrix, thus combining the high ionic conductivity and electrochemical stability that characterise ILs with the ease of processing associated with polymers. High ionic conductivity and electrochemical stability are both crucial parameters, the former allowing fast transistor switching by rapid ion diffusion upon application of a voltage at the gate electrode, and the latter permitting the application of high enough gate voltages without detrimental electrochemical reactions that could degrade device performance or even destroy the transistor. This has been shown to happen in OTFTs gated with hygroscopic polyelectrolytes that necessitate air moisture in order to show appreciable ionic conductivity, in which water electrolysis limits the applicable voltage range and can lead to high leakage currents. ${ }^{14,15}$ Previous reports of ion gels used as gate insulator in OTFTs include mixtures of an ionic liquid with triblock copolymers, ${ }^{16}$ with the fluorinated elastomer poly(vinylidenefluorideco-hexafluoropropylene), ${ }^{17}$ as well as with poly(ethylene oxide) obtained from a UV-crosslinkable diacrylated 

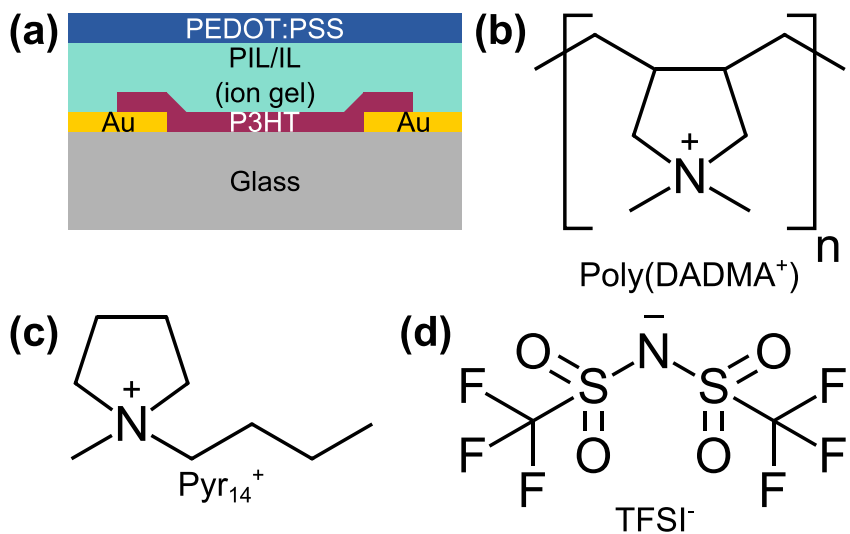

FIG. 1. (a) Schematic representation of the device geometry. Chemical structure of (b) the poly $\left(\mathrm{DADMA}^{+}\right.$) polycation, (c) the $\mathrm{Pyr}_{14}^{+}$cation and (d) the $\mathrm{TFSI}^{-}$anion.

monomer precursor. ${ }^{18}$ Poly(ionic liquid) (PILs), which are polyelectrolytes synthesised by direct polymerisation of an ionic liquid, ${ }^{19,20}$ are another class of materials that have not been extensively investigated as insulator materials for OTFTs. ${ }^{21,22}$ While they retain some of the advantages of ILs, such as electrochemical stability, they exhibit ionic conductivity values between that of ILs and conventional polyelectrolytes. However, PILs make very good host materials for Ils, as the electrochemical stability of the IL is preserved within the resulting gel and it ensures maximum chemical compatibility between the IL and its polymer matrix, therefore greatly reducing the risks of phase separation and leakage over long periods of time. PIL/IL blends also exhibit high ionic conductivities and favourable mechanical properties, even at moderate IL loading, and are easy to process. ${ }^{23}$

Here, we report the fabrication of OTFTs gated with a blend of the IL 1-Butyl-1-methylpyrrolidinium bis(trifluoromethylsulfonyl)imide $\quad\left(\mathrm{Pyr}_{14}\right.$ :TFSI) and the PIL poly(diallyldimethylammonium bis(trifluoromethylsulfonyl)imide)

(poly(DADMA:TFSI)) (Figures 1.b-d). The Mecerreyes group previously worked with these two materials and reported that they could be blended with a lithium salt for battery applications. ${ }^{24,25}$ They showed that blends containing these two materials in $50: 50 \mathrm{w} / \mathrm{w}$ proportions form free-standing membranes when cast from solution. They extensively characterised the resulting ion gel and found that it is thermally stable up to $300{ }^{\circ} \mathrm{C}$ , that it does not exhibit any signs of leakage or phase separation after prolonged storage times and that it has a wide electrochemical stability window of $7 \mathrm{~V}$. The ionic conductivity of the PIL/IL ion gel was measured to be $0.3 \mathrm{mS} \mathrm{cm}^{-1}$ at $23{ }^{\circ} \mathrm{C}$, which is between that of poly(DADMA:TFSI) $\left(0.007 \mathrm{mS} \mathrm{cm}{ }^{-1}\right)$ and Pyr $_{14}$ :TFSI $\left(3 \mathrm{mS} \mathrm{cm}^{-1}\right)$.

The devices are also based on the polymer semiconductor poly(3-hexylthiophene) (P3HT), which typically exhibits moderate mobilities around $0.1 \mathrm{~cm}^{2} \mathrm{~V}^{-1} \mathrm{~s}^{-1} \cdot 26$
Besides improving the semiconductor mobility, it is possible to make high performance OTFTs by maximising the other parameters affecting the transconductance

$$
g_{m}=\frac{\partial I_{D}}{\partial V_{G}}=\frac{W}{L} \mu C_{i}\left(V_{G}-V_{t}\right)
$$

where $I_{D}$ is the drain current, $V_{G}$ is the gate voltage, $V_{t}$ is the threshold voltage, $W$ and $L$ are the channel width and length, respectively, $\mu$ is the semiconductor hole mobility and $C_{i}$ is the effective insulator capacitance per unit area. Therefore, it is possible to obtain a large transconductance when an ion gel insulator with a large effective capacitance is used, as described above. Besides the parameters directly affecting the transconductance, parasitic effects such as leakage currents and hysteresis must be minimised. These can be particularly prominent in electrolyte-gated OTFTs, due to sluggish ionic diffusion effects. ${ }^{27}$

As represented in figure 1.a, the devices were fabricated in air on a glass substrate and bottom Au source and drain electrodes were defined by photolithography, forming a channel of width $W=2000 \mu \mathrm{m}$ and length $L=10 \mu \mathrm{m}$, resulting in a reasonably large $W / L$ ratio of 200. P3HT was then spin-coated at $2000 \mathrm{rpm}$ from a $5 \mathrm{mg} \mathrm{mL}-1$ solution in 1,2-dichlorobenzene in order to form thin-films of thickness $\approx 20 \mathrm{~nm}$. Thicker films were observed to result in slow switching, higher off currents and increased hysteresis, consistent with previous reports. ${ }^{28}$ Similar parasitic effects were observed when the semiconductor film was not patterned to cover the channel area only, which we attribute to ionic penetration over the entire volume of the film that is covered by the electrolyte. P3HT was therefore patterned via photolithography using the commercially available fluorinated photoresist OSCoR 4001 (Orthogonal Inc.). Fluorinated photoresists and solvents have been shown to not interact with organic materials, allowing fine patterning without affecting their structural and electrical characteristics. ${ }^{29}$ The ability to pattern organic semiconductors with photolithography means that this widespread patterning method, currently used at industrial scale for mass production of integrated circuits, can be applied to large-area, high throughput, high-definition and low-cost processing of organic electronic devices. Printing methods are another option to pattern organic semiconductors, however, these rarely offer a satisfying trade-off between high-throughput and high-resolution, and significant development of the various technologies is still required. For example, inkjet printing can be used to pattern features around $10 \mu \mathrm{m}$, albeit at low speed and on small areas, while gravure, flexography and offset printing are very high speed, large-area techniques that typically achieve resolutions of $100 \mu \mathrm{m} .{ }^{30}$ Once the polymer semiconductor was deposited and patterned, the PIL/IL ion gel was drop-cast over the channel area from an acetone solution, followed by drop-casting of the conducting polymer poly(3,4-ethylenedioxythiophene)poly(styrenesulfonate) (PEDOT:PSS) mixed with 10 

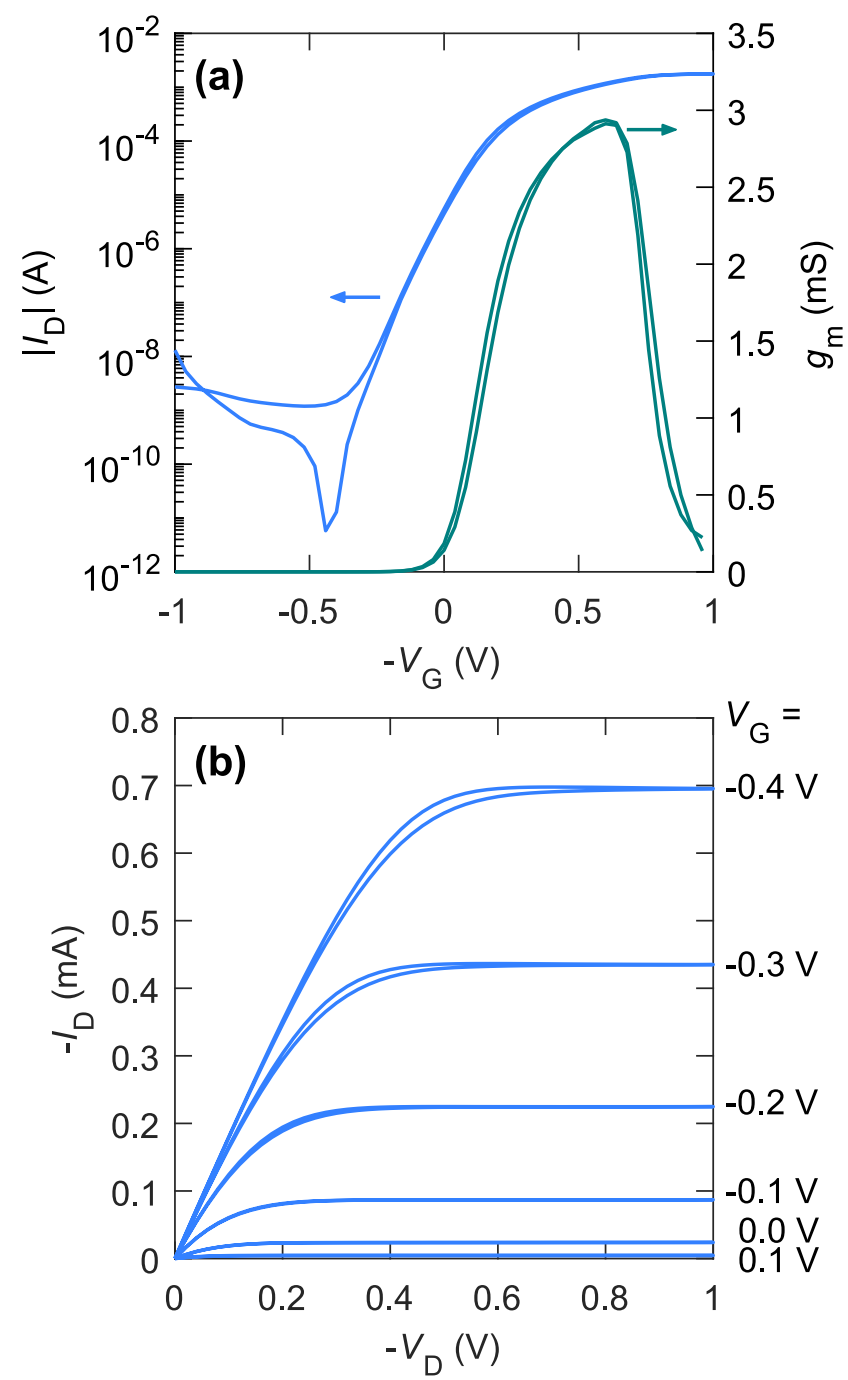

FIG. 2. Typical electrical characteristics of an ion gel-gated OTFT. (a) Transfer characteristics obtained under a drain voltage $V_{D}=-1 \mathrm{~V}$ and with a sweep rate of $40 \mathrm{mV} \mathrm{s}^{-1}$, as well as the corresponding transconductance $g_{m}$. (b) Output characteristics.

vol\% ethylene glycol as the top gate electrode and drying at $100{ }^{\circ} \mathrm{C}$ for $5 \mathrm{~min}$. The thick and porous PEDOT:PSS gate electrode ensures a large effective gate area, resulting in a large voltage drop at the gel/semiconductor interface, which maximises channel current modulation. ${ }^{31}$

Figures 2.a and 2.b show the typical output and transfer characteristics of a device, which were obtained under inert nitrogen atmosphere. Both characteristics exhibit only a minor hysteresis, which we attribute to the various design choices described above and to the high ionic conductivity of the PIL/IL ion gel. The output curve shows very distinct and well-defined linear and saturation regions, with the saturation drain current $I_{D \text {, sat }}$ being virtually constant as a function of the drain voltage $V_{D}$. This last observation suggests a negligible leakage current through the gel in the saturation regime. Furthermore, the linearity of the curves at low $V_{D}$ indicates

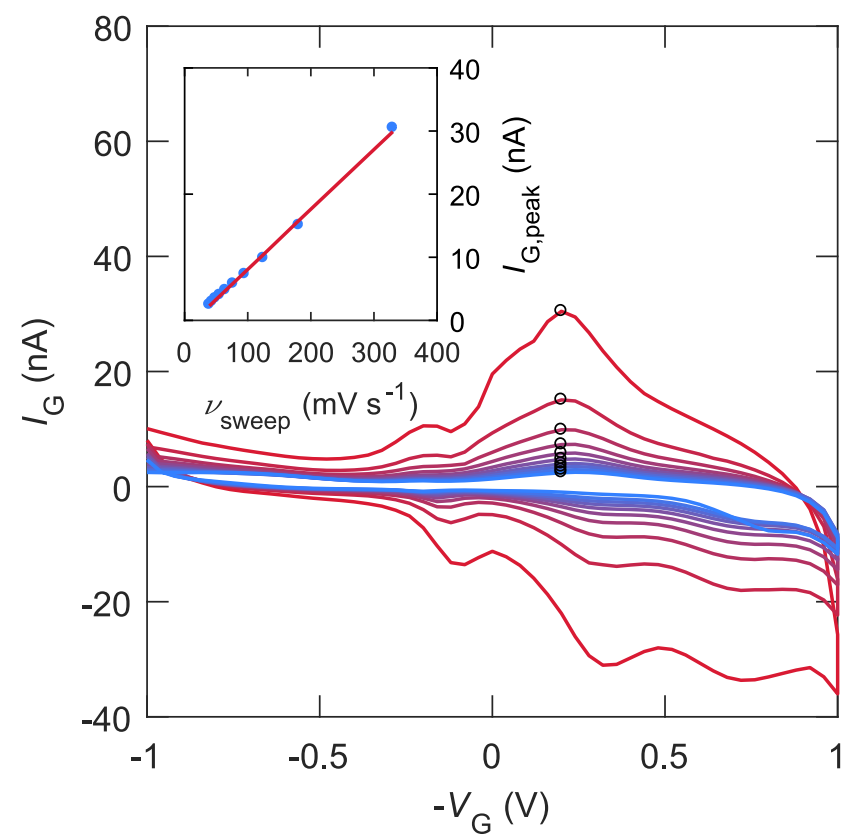

FIG. 3. Evolution of the gate current $I_{G}$ as a function of the gate voltage $V_{G}$ at various sweep rates. The black circles indicates the peak gate current values used for the capacitance measurement. The inset shows the peak gate current $I_{G, p e a k}$ as a function of the sweep rate $\nu_{\text {sweep }}$. The red line is a linear fit to the data.

that the channel current is not limited by contact resistance, despite the bottom contact configuration used here. This is in agreement with previous reports showing that contact resistance is much lower in ion gel-gated OTFTs, with regards to conventional gate dielectrics. ${ }^{32}$ The cause of this observation is still unclear, but it has been suggested that it could be the result of an additional electric field arising from the presence of ions near the contacts, or that the contact resistance could be reduced as a result of electrochemical doping. The transfer characteristics reveals a good on/off ratio of $c a .10^{6}$ and a very high on-current $I_{D \text {, on }}>1 \mathrm{~mA}$ at $V_{G}=-1 \mathrm{~V}$. This is the result of the very high transconductance of the device, which is shown in figure 2.a. It reaches a maximum value $g_{m, \max }=2.9 \pm 0.1 \mathrm{mS}$ (averaged over 9 devices), which is comparable to that of PEDOT:PSS depletionmode electrochemical transistors (OECTs) gated with an aqueous electrolyte, which possess some of the highest reported values to date. ${ }^{9}$ It can also be seen that the transconductance decreases sharply past this maximum and is close to zero at $V_{G}=-1 \mathrm{~V}$. In fact, at more negative gate voltages, the drain current starts decreasing and the transconductance becomes negative. This phenomenon was extensively studied and shown to occur even in single crystal organic semiconductors, and is attributed to strong coulombic interactions between the negatively charged ions and the positively charged holes at high carrier densities. ${ }^{33}$

In order to gain more insight into the high value of 


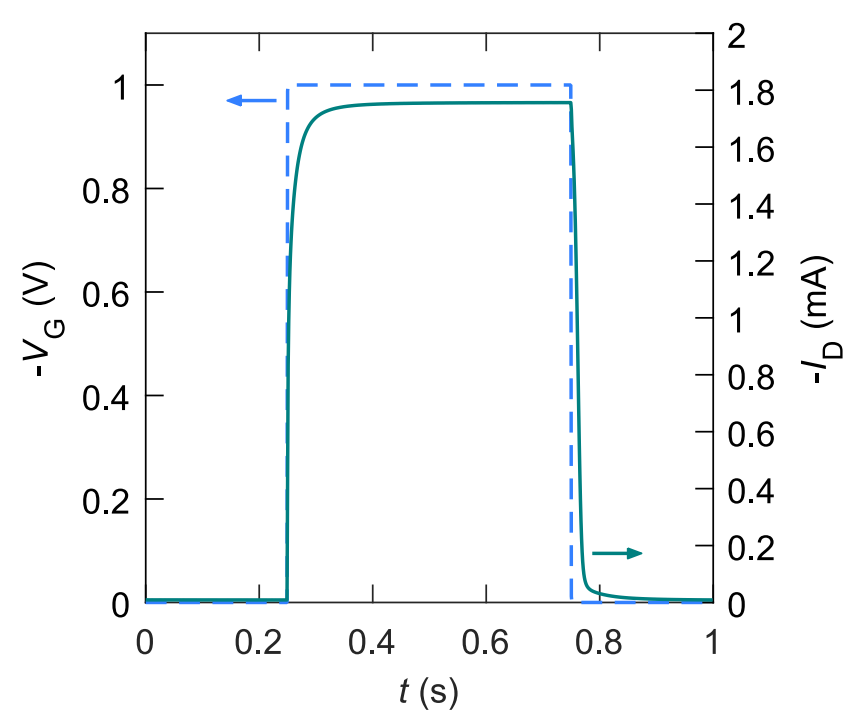

FIG. 4. Transient response of the drain current $I_{D}$ of the device submitted to a pulse of amplitude $V_{G}=-1 \mathrm{~V}$, while the drain voltage was kept at $V_{D}=-1 \mathrm{~V}$

$g_{m}$, we evaluated the effective capacitance of the device $C_{i}$ by measuring the displacement current -i.e. the gate current $I_{G}$ as a function of $V_{G}$ with the source and drain electrodes grounded -at various $V_{G}$ sweep rates $\nu_{\text {sweep }}$ (Figure 3.a). The device capacitance can then be evaluated using the relation $I_{G}=C \nu_{\text {sweep }}$, as shown in figure 3.b. Here, we obtained $C=79 \pm 7 \mathrm{nF}$. It was recently shown that the capacitance of OECTs is volumetric in nature. ${ }^{34,35}$ The implications are that $g_{m}$ is dependent on the thickness of the semiconductor and that the value of the effective capacitance per unit area $C_{i}$ used in equation 1 is a projected value of the capacitance per unit volume $C^{*}$ of the three-dimensional electrode defined by the bulk of the semiconductor channel, and not the interfacial double layer capacitance $C_{D L}=20$ $\mu \mathrm{F} \mathrm{cm}^{-2}$ of the PIL/IL ion gel, that we obtained using a $\mathrm{Au} /$ ion gel/PEDOT:PSS stack via impedance spectroscopy. Here, we obtained averages of $C_{i}=197 \pm 17 \mu \mathrm{F}$ $\mathrm{cm}^{-2}$ and $C^{*}=98 \pm 9 \mu \mathrm{F} \mathrm{cm}{ }^{-3}$. These large capacitance values explain how such a large transconductance could be obtained, even with a thin semiconductor film only $20 \mathrm{~nm}$-thick. We also note that an accurate evaluation of these capacitance values could not have been obtained without a reliable high-resolution patterning process for the semiconductor film such as the othogonal patterning method employed here. Finally, using $C_{i}$ and fitting the linear part of $\sqrt{I_{D}}$ as a function of $V_{G}$, we obtained an average mobility value $\mu=0.16 \pm 0.01 \mathrm{~cm}^{2} \mathrm{~V}^{-1} \mathrm{~s}^{-1}$, a reasonably high value for $\mathrm{P} 3 \mathrm{HT}$, as well as a small threshold voltage $V_{t}=0.06 \pm 0.02 \mathrm{~V}$.

Then, we investigated the transient response of the device to a pulse of amplitude $V_{G}=-1 \mathrm{~V}$, as displayed in figure 4 . The corresponding rise and fall times (taken at $90 \%$ of the final response) were $18 \pm 7 \mathrm{~ms}$ and $20 \pm 6 \mathrm{~ms}$, respectively. This corresponds to an operating frequency of about $50 \mathrm{~Hz}$. Relatively slow switching times $(<1 \mathrm{kHz})$ are typical for unoptimised electrolyte-gated OTFTs limited by ionic diffusion. $6,36,37$ A straightforward way to improve the operating frequency of the devices would be to use a different PIL/IL system with a higher ionic conductivity, or that allows for a higher IL content without loss of the mechanical properties of the resulting ion gel. Pushing the IL content in the current system to $75 \mathrm{wt} \%$ resulted in a slight improvement in switching speed (around $10 \mathrm{~ms}$ ), at the cost of the gel's mechanical properties, making the deposition of the PEDOT:PSS gate electrode difficult and resulting in a high number of short-circtuited devices and larger disparities between the characteristics of working devices. Besides, much faster switching up to $10 \mathrm{kHz}$ was previously demonstrated by reducing the area of the source and drain contacts, therefore reducing the parasitic overlap capacitance between the gel and the contacts, ${ }^{38}$ suggesting that the factor truly limiting device speed is the current device geometry. While the large channel width $W=2000$ $\mu \mathrm{m}$ is desirable in order to keep the $W / L$ ratio to a high value (see equation 1 ), it considerably increases the contact area. Thus, there is scope for improvement regarding the dynamic behaviour of the device. Using nanopatterning methods such as nano-imprint lithography ${ }^{39}$ or electron beam lithography ${ }^{40}$ could be useful to explore the characteristics of the devices with a reduced footprint (smaller channel and electrode surface areas), while keeping a large $W / L$ ratio. It was also previously reported that electrolyte-gated OTFTs with nanoscale channels perform better than OTFTs gated with conventional dielectrics, thanks to a much higher transverse electric field that limits undesirable short channel effects, which might render the operation of ion gel-gated organic nano-transistors possible. ${ }^{41}$

Finally, in order to assess the stability of the device and ensure that the doping process is reversible, the gate electrode was driven by a square wave signal oscillating between $V_{G}=0 \mathrm{~V}$ and $-1 \mathrm{~V}$ at a frequency of $0.5 \mathrm{~Hz}$ while $V_{D}=-1 \mathrm{~V}$ and the resulting drain current response was recorded (see figure $\mathrm{S} 1$, supplementary material). The relative decrease in on-current was only $1.8 \%$ after 1000 cycles, or more than $2000 \mathrm{~s}$ (about 35 minutes). These results suggest that the electrochemical reaction associated with the doping process does not induce any dramatic degradation of the semiconductor or ion gel within the operational voltage window used.

In conclusion, we demonstrated high performance OTFTs employing a PIL/IL ion gel as gate insulator. Thanks to the very high effective capacitance of about $200 \mu \mathrm{F} \mathrm{cm}^{-2}$ resulting from three-dimensional ionic doping of the semiconductor, the devices exhibit a very high transconductance of $3 \mathrm{mS}$ and channel currents higher than $1 \mathrm{~mA}$ at operating voltages of only $-1 \mathrm{~V}$. The use of orthogonal photolithography to pattern a thin polymer semiconductor channel resulted in minimised hysteresis and leakage currents, leading to a good on/off ratio around $10^{6}$. The devices also appeared to be stable 
upon repeated switching and no signs of significant degradation from the doping process were observed within the operating voltage range used. Study of the dynamic behaviour revealed the limitation of the deposition method by simple drop-casting of the ion gel $(50 \mathrm{~Hz}$ dynamic frequency) and the device switching speed will be an area of focus for future optimisation. Nonetheless, the static characteristics are more than satisfying for a variety of applications, such as sensing or driving organic light-emitting diodes, where a high transconductance is required.

\section{SUPPLEMENTARY MATERIAL}

See supplementary material for a plot showing the device stability over a large number of switching cycles.

\section{ACKNOWLEDGEMENTS}

This work was supported by the European Commission's 7th Framework Programme (FP7/2007-2013) under grant agreement no. 607896 (OrgBIO).

${ }^{1}$ L. Kergoat, B. Piro, M. Berggren, G. Horowitz, and M. C. Pham, Analytical and Bioanalytical Chemistry 402, 1813 (2012).

${ }^{2}$ G. Tarabella and F. Mohammadi, Chemical Science 4, 1395 (2013).

${ }^{3}$ S. H. Kim, K. Hong, W. Xie, K. H. Lee, S. Zhang, T. P. Lodge, and C. D. Frisbie, Advanced Materials 25, 1822 (2013).

${ }^{4}$ E. Said, X. Crispin, L. Herlogsson, S. Elhag, N. D. Robinson, and M. Berggren, Applied Physics Letters 89, 143507 (2006).

${ }^{5}$ S. Ono, K. Miwa, S. Seki, and J. Takeya, Applied Physics Letters 94, 063301 (2009)

${ }^{6}$ B. D. Nilsson, M. Chen, T. Kugler, T. Remonen, M. Armgarth, and M. Berggren, Advanced Materials 14, 51 (2002).

${ }^{7}$ J. D. Yuen, A. S. Dhoot, E. B. Namdas, N. E. Coates, M. Heeney, I. McCulloch, D. Moses, and A. J. Heeger, Journal of the American Chemical Society 129, 14367 (2007).

${ }^{8}$ O. Larsson, A. Laiho, W. Schmickler, M. Berggren, and X. Crispin, Advanced Materials 23, 4764 (2011).

${ }^{9}$ D. Khodagholy, J. Rivnay, M. Sessolo, M. Gurfinkel, P. Leleux, L. H. Jimison, E. Stavrinidou, T. Herve, S. Sanaur, R. M. Owens, and G. G. Malliaras, Nature communications 4, 2133 (2013).

${ }^{10} \mathrm{~J}$. Rivnay, R. M. Owens, and G. G. Malliaras, Chemistry of Materials 26, 679 (2014).

${ }^{11}$ S. P. White, K. D. Dorfman, and C. D. Frisbie, Analytical Chemistry 87, 1861 (2015).

${ }^{12}$ D. Braga, N. C. Erickson, M. J. Renn, R. J. Holmes, and C. D. Frisbie, Advanced Functional Materials 22, 1623 (2012).

${ }^{13}$ Y.-S. Ye, J. Rick, and B.-J. Hwang, J. Mater. Chem. A 1, 2719 (2013).

${ }^{14}$ E. Said, O. Larsson, M. Berggren, and X. Crispin, Advanced Functional Materials 18, 3529 (2008).
${ }^{15}$ Q. Thiburce and A. J. Campbell, Advanced Electronic Materials 3, 1600421 (2017)

${ }^{16}$ J. H. Cho, J. Lee, Y. He, B. Kim, T. P. Lodge, and C. D. Frisbie, Advanced Materials 20, 686 (2008).

${ }^{17}$ K. H. Lee, M. S. Kang, S. Zhang, Y. Gu, T. P. Lodge, and C. D. Frisbie, Advanced Materials 24, 4457 (2012).

${ }^{18}$ S. W. Lee, B. S. Kim, J. J. Park, J. H. Hur, J. M. Kim, T. Sekitani, T. Someya, and U. Jeong, Journal of Materials Chemistry 21, 18804 (2011).

${ }^{19}$ D. Mecerreyes, Progress in Polymer Science (Oxford) 36, 1629 (2011).

${ }^{20}$ J. Yuan, D. Mecerreyes, and M. Antonietti, Progress in Polymer Science 38, 1009 (2013).

${ }^{21}$ M. Hamedi, L. Herlogsson, X. Crispin, R. Morcilla, M. Berggren, and O. Inganäs, Advanced Materials 21, 573 (2009).

${ }^{22}$ J. H. Choi, W. Xie, Y. Gu, C. D. Frisbie, and T. P. Lodge, ACS Applied Materials \& Interfaces 7, 7294 (2015).

${ }^{23}$ A. S. Shaplov, R. Marcilla, and D. Mecerreyes, Electrochimica Acta 175, 18 (2015).

${ }^{24}$ A. L. Pont, R. Marcilla, I. De Meatza, H. Grande, and D. Mecerreyes, Journal of Power Sources 188, 558 (2009).

${ }^{25}$ G. B. Appetecchi, G. T. Kim, M. Montanino, M. Carewska, R. Marcilla, D. Mecerreyes, and I. De Meatza, Journal of Power Sources 195, 3668 (2010).

${ }^{26}$ J. F. Chang, B. Sun, D. W. Breiby, M. M. Nielsen, T. I. Sölling, M. Giles, I. McCulloch, and H. Sirringhaus, Chemistry of Materials 16, 4772 (2004).

${ }^{27}$ Y. Xia, J. Cho, B. Paulsen, C. D. Frisbie, and M. J. Renn, Applied Physics Letters 94, 013304 (2009).

${ }^{28}$ S. H. Kim, K. Hong, K. H. Lee, and C. D. Frisbie, ACS Applied Materials \& Interfaces 5, 65806585 (2013).

${ }^{29}$ A. A. Zakhidov, J. K. Lee, H. H. Fong, J. A. DeFranco, M. Chatzichristidi, P. G. Taylor, C. K. Ober, and G. G. Malliaras, Advanced Materials 20, 3481 (2008).

${ }^{30}$ V. Subramanian, J. Cen, A. de la Fuente Vornbrock, G. Grau, H. Kang, R. Kitsomboonloha, D. Soltman, and H.-Y. Tseng, Proceedings of the IEEE 103, 567 (2015).

${ }^{31}$ O. Yaghmazadeh, F. Cicoira, D. a. Bernards, S. Y. Yang, Y. Bonnassieux, and G. G. Malliaras, Journal of Polymer Science, Part B: Polymer Physics 49, 34 (2011).

${ }^{32}$ D. Braga, M. Ha, W. Xie, and C. D. Frisbie, Applied Physics Letters 97, 193311 (2010).

${ }^{33}$ W. Xie, F. Liu, S. Shi, P. P. Ruden, and C. D. Frisbie, Advanced Materials 26, 2527 (2014).

${ }^{34}$ J. Rivnay, P. Leleux, M. Ferro, M. Sessolo, A. Williamson, D. A. Koutsouras, D. Khodagholy, M. Ramuz, X. Strakosas, R. M. Owens, C. Benar, J.-M. Badier, C. Bernard, and G. G. Malliaras, Science Advances 1, 1400251 (2015).

${ }^{35}$ C. M. Proctor, J. Rivnay, and G. G. Malliaras, Journal of Polymer Science, Part B: Polymer Physics 54, 1433 (2016).

${ }^{36}$ M. J. Panzer and C. D. Frisbie, Journal of the American Chemical Society 129, 6599 (2007).

${ }^{37}$ J. Lee, L. G. Kaake, H. J. Cho, X. Y. Zhu, T. P. Lodge, and C. D. Frisbie, Journal of Physical Chemistry C 113, 8972 (2009).

${ }^{38}$ F. Zare Bidoky and C. D. Frisbie, ACS Applied Materials and Interfaces 8, 27012 (2016).

${ }^{39}$ S. G. Higgins, B. V. O. Muir, J. Wade, J. Chen, B. Striedinger, H. Gold, B. Stadlober, M. Caironi, J.-S. Kim, J. H. G. Steinke, and A. J. Campbell, Advanced Electronic Materials 1, 1500024 (2015).

${ }^{40}$ G. S. Tulevski, C. Nuckolls, A. Afzali, T. O. Graham, and C. R. Kagan, Applied Physics Letters 89, 183101 (2006).

${ }^{41}$ L. Herlogsson, Y. Y. Noh, N. Zhao, X. Crispin, H. Sirringhaus, and M. Berggren, Advanced Materials 20, 4708 (2008). 